\title{
Bulgakova Tatiana, Nanai Shamanic Culture in
} Indigenous Discourse

Fürstenberg/Havel, Kulturstiftung Sibirien, 2013, 261 p. ISBN

978-3-942883-14-6

Aurore Dumont et Alexandra Lavrillier

\section{(2) OpenEdition}

Journals

Édition électronique

URL : https://journals.openedition.org/emscat/2694

DOI : $10.4000 /$ emscat.2694

ISSN : 2101-0013

Éditeur

Centre d'Etudes Mongoles \& Sibériennes / École Pratique des Hautes Études

Référence électronique

Aurore Dumont et Alexandra Lavrillier, «Bulgakova Tatiana, Nanai Shamanic Culture in Indigenous Discourse », Études mongoles et sibériennes, centrasiatiques et tibétaines [En ligne], 46 | 2015, mis en ligne le 10 septembre 2015, consulté le 13 juillet 2021. URL : http://journals.openedition.org/emscat/ 2694 ; DOI : https://doi.org/10.4000/emscat.2694

Ce document a été généré automatiquement le 13 juillet 2021.

(c) Tous droits réservés 


\section{Bulgakova Tatiana, Nanai Shamanic Culture in Indigenous Discourse}

Fürstenberg/Havel, Kulturstiftung Sibirien, 2013, 261 p. ISBN

978-3-942883-14-6

Aurore Dumont et Alexandra Lavrillier

\section{RÉFÉRENCE}

Bulgakova Tatiana, Nanai Shamanic Culture in Indigenous Discourse, Fürstenberg/Havel, Kulturstiftung Sibirien, 2013

1 L'ouvrage de Tatiana Bulgakova est consacré aux Nanaï (nanaitsy) ${ }^{1}$ qui vivent sur les rives des fleuves Amour et Oussouri dans l'Extrême-Orient russe, et aux discours que ce peuple toungouse tient sur ses pratiques chamaniques et sur les dires et gestes des esprits. Composé de quatre parties complétées par des photographies, un index et un glossaire, il progresse selon une logique thématique, l'auteur exposant le résultat de ses recherches menées sur trois décennies (1980-2012).

Dans la première partie, intitulée "Research into shamanic culture's mysteries", Tatiana Bulgakova montre comment l'ethnographie du chamanisme est profondément influencée par des facteurs subjectifs. Les données récoltées se révèlent parfois lacunaires, contradictoires, voire absentes. En effet, le chercheur de terrain est confronté à diverses interprétations résultant d'expériences individuelles multiformes chez les informateurs. Afin de se prémunir contre d'éventuelles représailles de l'ennemi, les chamanes dissimulent fréquemment toute information relative aux combats entre chamanes. Or, comme le souligne Bulgakova, l'absence d'informations ne signifie pas pour autant l'inexistence du phénomène. C'est ainsi que certaines notions - le réceptacle immatériel des âmes des patients (dëkaso) et les territoires chamaniques (gora et dërgil) - ne sont apparues dans la littérature ethnographique que dans les années 1990, sous l'impulsion d'Anna V. Smoliak, (ethnographe soviétique, spécialiste des Toungouses de l'Amour, dont les Nanaï). Les informations, contradictoires au 
premier abord, contraignent le chercheur à clarifier des interprétations qui varient selon le contexte : par exemple, l'automutilation que s'inflige le chamane peut être une démonstration de ses talents chamaniques, une bataille contre un ennemi, ou encore une offrande aux esprits.

La deuxième partie, "Rivalry in shamanic healing ", aborde les troubles collectifs et leur interprétation par les Nanaï. Contrairement à une idée répandue, la «maladie chamanique » et son éventuelle guérison (que l'auteur préfère appeler rémission) n'affectent pas uniquement le chamane, mais l'ensemble de son groupe de parenté. Des contacts rapprochés avec le chamane entrainent une sensibilité aux esprits chez ses proches parents. Certains en viennent même à chamaniser dans leurs rêves ou dans la vie réelle pour leurs besoins personnels, sans pour autant être reconnus comme chamanes par la société.

4 Par ailleurs, maladie et guérison sont liées à ce que les néo chamanes appellent nikheleuri, terme nanaï signifiant « ouvrir », c'est-à-dire "l'ouverture d'un individu aux esprits ». Si la maladie affectant une personne est occasionnée par l'installation d'un esprit dans son corps, le traitement consiste à l'expulser. En revanche, si l'âme (panian) d'un individu a quitté son corps, le chamane doit la localiser puis la déposer dans le réceptacle immatériel, dëkaso pratique dont seuls les "non-chamanes" peuvent bénéficier. Selon le discours autochtone, le dëkaso s'apparente à une maison, une ville, ou plus généralement à une prison. En effet, les âmes emprisonnées tentant de s'échapper sont nourries par les esprits en échange de guérison.

5 À l'inverse, l' «initiation» chamanique se conclut par la disparition des âmes humaines de celui qui devient chamane et sont remplacées par des esprits chamaniques (seven). L'« initiation » n'est pas la phase finale de la formation d'un chamane: ses contacts avec les esprits nécessitent d'être renouvelés, d'où l'existence de «multiples initiations ».

6 Enfin, l'auteur analyse les critères d'évaluation de la puissance d'un chamane. Selon la vision autochtone, celle-ci ne repose pas seulement sur la capacité de guérir un patient ou l'aptitude à produire des miracles. Les chamanes se mesurent entre eux grâce à l'arbre chamanique établi dans le monde des esprits: le chamane peut déterminer la puissance de chacun de ses adversaires selon la place qu'il occupe sur une branche et selon que celle-ci est plus ou moins haute. Lorsqu'un chamane parle de lui-même à un non-chamane, il fait valoir son intrépidité face aux esprits mais face à un autre chamane, il se déprécie, de peur que celui-ci, toujours tenu pour être un adversaire en puissance, ne lui vole ses esprits seven. Enfin, si les rumeurs sont une composante essentielle dans l'élaboration de l'opinion publique sur le pourvoir réel ou supposé d'un chamane, les "gens ordinaires" ne critiquent jamais ouvertement les chamanes sous peine d'attiser la colère des esprits.

7 La troisième partie, intitulée «Shamanising by arts ", est consacrée à la pratique chamanique par les arts (au sens large), autrement dit à la littérature orale (contes, récits épiques, etc.) et aux danses anciennes et contemporaines. Chaque récit, qui est vu comme une "route », est une réalité passée, transformée en conte par un chamane qui l'aura reçu en rêve, puis transmis à la communauté. Conteur comme chamane content, à cette différence que le premier connaît la " route ", tandis que le second est guidé par ses esprits en territoire inconnu. Pour tous deux, conter est un moyen de rester liés aux esprits. 
8 L'auteur relève de la cruauté dans certains récits. Les sources en sont variées, les esprits seven, hérités de chamane en chamane, sont chargés d'une agressivité accumulée qu'il convient d'atténuer. Les gens ordinaires peuvent également être animés par des seven agressifs, notamment les esprits « amants", jaloux des époux. De la violence peut naître de la cohabitation entre un humain et un esprit (il faut alors soumettre l'esprit), ou bien du mariage entre deux humains, dont l'un, au moins, est chamane. Une femme chamane est susceptible de voler le seven plus puissant d'un homme (notamment par le biais de relations sexuelles), mais non l'inverse; le mariage peut aussi permettre une paix temporaire entre deux clans de chamanes ennemis. La future mariée et les amants esprits sont une représentation constante dans les contes nanaï, où comme dans les pratiques chamaniques, les jeunes gens servent d'appât pour entrer en contact avec les esprits. Lorsqu'une mariée rejoint la famille de son époux, elle y apporte les esprits de son propre clan, potentiellement dangereux pour la famille de son mari, qui, pour les écarter, tire des coups de feu durant la cérémonie nuptiale.

Dans la société nanaï traditionnelle, toutes les danses sont des offrandes aux esprits. Les mouvements du corps accompagnés du rythme des tambours et des chants rendent les esprits actifs et permettent d'établir un lien entre les humains et eux. Lors des rituels, les participants puis le chamane dansent à tour de rôle, utilisant tambour et ceinture chamaniques. Ce sont les esprits seven qui apprennent aux humains à danser lors des rituels.

10 Pendant la décennie de transition (1985 et 1995) entre le chamanisme dit classique (aujourd'hui disparu) et le néo-chamanisme, apparaît un nouveau phénomène consistant à exhiber les chamanes, les chanteurs et les conteurs lors de spectacles publics. Qu'ils soient à but rituel ou non, ces spectacles soumettent les spécialistes rituels aux représailles de leurs esprits auxiliaires ainsi convoqués. En effet, il est arrivé, dit-on, que des esprits n'ayant reçu aucune contrepartie, se vengent, causant ainsi la mort de plusieurs chamanes. Actuellement, dans le contexte du renouveau ethnique, des pratiques chamaniques sont réinventées - de manière artificielle dit l'auteur - sur la base de matériaux ethnographiques locaux, mais aussi pan-sibériens et internationaux. Aujourd'hui, afin d'éviter les représailles des esprits, les acteurs jouant des rôles de chamanes évitent soigneusement d'appeler les esprits: ils ne prononcent pas les mots chamaniques appropriés et portent de faux costumes; d'autres se considérant chamanes, utilisent la scène et le contact avec les esprits pour « soigner » l'auditoire. Selon l'auteur, il est vain de faire renaître le chamanisme nanaï traditionnel ainsi.

11 La dernière partie, « Political and legal labours ruled by shamanic spirits » examine les aspects juridiques et politiques du chamanisme. Certains auteurs russes considéraient la cour traditionnelle des Nanaï comme démocratique. Mais pour les Nanaï, le jugement final provenait des esprits, l'autorité juridique étant autrefois reliée au chamanisme, et par conséquent cachée à l'observateur. Selon l'autorité juridique nanaï, le jugemédiateur (diangnian) avait pour rôle de désamorcer les disputes entre clans. La plupart $\mathrm{du}$ temps, les parents du fautif faisaient appel à lui non pas pour punir le fauteur de troubles mais pour neutraliser le conflit et l'indignation des victimes. Le jugemédiateur et le chamane partageaient de nombreux points communs: tous deux disaient voir leurs esprits en rêve et devaient surmonter un épisode de maladie avant d'être élus. 
12 La fin de la dernière partie de l'ouvrage s'achève sur les relations entre le pouvoir soviétique et les chamanes. De nouveau, l'auteur rapporte certains faits habituellement passés sous silence. Si pendant la période soviétique, le chamane était considéré comme un ennemi de classe, les chefs d'accusation n'étaient pas toujours de nature religieuse. L'auteur cite l'exemple du chamane Bogdan Onenko. Sous l'influence de la propagande athéiste, nombre de ses patients refusèrent de le rétribuer en animaux sacrifiés, promis par le chamane à ses esprits en échange de guérison. Le non-paiement entraîna la vengeance des esprits, le chamane et ses patients tombant malade un à un. Afin de rétablir l'ordre, Bogdan Onenko fut obligé de voler les animaux de ses patients pour satisfaire ses esprits. Ce larcin lui valut une arrestation non pas pour pratique illégale du chamanisme mais pour vol.

Bulgakova insiste sur une autre croyance rarement évoquée : les chamanes étaient certes persécutés par le régime soviétique, mais disaient l'être encore plus par leurs esprits qui les forçaient à continuer de chamaniser. Certains chamanes ont répondu à l'appel, pratiquant avec des casseroles ou des conserves en guise de tambours. En outre, d'autres ont bénéficié d'une permission officielle de chamaniser après avoir guéri des membres du gouvernement. L'auteur révèle par ailleurs que les "persécuteurs antichamanes » étaient plus souvent des Nanaï que des Russes. Enfin, certains Nanaï partis étudier à Moscou offraient des sacrifices à l'esprit Staline: voilà comment cinq informateurs de l'auteur étaient à la fois chamanes et communistes !

L'ouvrage de Tatiana Bulgakova est remarquable. Il se distingue d'abord par la richesse des données ethnographiques recueillies en langue vernaculaire, à une époque où les chamanes étaient encore nombreux. L'auteur a pu établir des relations de grande confiance avec les chamanes, tâche particulièrement ardue. Il se caractérise ensuite par l'importance accordée au discours autochtone: les propos des chamanes et de la population nanaï (qui sont, selon Bulgakova, les « détenteurs de la tradition ») viennent systématiquement soutenir les propos de l'auteur. Il convient ainsi de souligner l'honnêteté scientifique de Tatiana Bulgakova et son sens moderne de l'éthique du terrain, qui questionne l'opposition « observant-observé » et reconnait à l'informateur le rôle qu'il remplit réellement, soit celui d'« assistant ». L'auteur a en outre multiplié les terrains afin d'enrichir et de réinterpréter les données récoltées. Enfin, l'originalité des thématiques, la précision de leur analyse et la mise en perspective de pratiques peu connues confèrent une grande valeur à l'ouvrage.

Cependant, le lecteur peine parfois à établir un lien chronologique entre ce qui existe aujourd'hui et ce qui a disparu. À cet égard, une analyse approfondie du néochamanisme aurait été la bienvenue. Il est également regrettable que certaines informations de première importance soient reléguées en note de bas de pages et qu'aucune mention ne soit faite du mode de vie traditionnel des Nanaï. Ces quelques remarques n'enlèvent toutefois rien à la qualité et au caractère novateur de cet ouvrage de premier plan sur le chamanisme sibérien. 


\section{NOTES}

1. Environ cinq mille Nanaï vivent en Chine sur les rives des fleuves Oussouri et Soungari dans la province du Heilongjiang. Dans la classification officielle chinoise, ils sont désignés sous l'appellation Hezhe et font partie des « minorités ethniques ». 